\title{
RIEMANN SURFACES AND SMALL POINT SETS
}

\section{LARS V. AHLFORS}

I have been asked to talk about Nevanlinna's contributions to Riemann surfaces and the related subject of small point sets in function theory. What I shall try to do is to make an effort to understand how Nevanlinna came to Riemann surfaces in the first place and to make a factual assessment of what he did and of its impact on contemporary and later work in the same field. I want to prepare you that I shall not shy away from a certain amount of criticism when I think it furthers my purpose, but please keep in mind that I am speaking from a safe distance of hindsight, and that my critique is not intended to detract from the value of Nevanlinna's pioneering work. It is not quite easy to draw the right line, for there were many persons involved and it is difficult to tell who was influenced by whom.

Riemann surfaces were not Nevanlinna's first love. In his younger years he was exclusively interested in functions on a plane region. Nevanlinna's fame came early and it was principally based on his theory of meromorphic functions and clever use of potential theory which he had started together with his brother Frithiof and then developed further to the powerful method of harmonic measure.

Even for these topics Riemann surfaces were needed, above all in the context of uniformization, but he did not yet study Riemann surfaces for their own sake. He could of course not help being interested in the Riemann surfaces that occur as the image of an entire or meromorphic function. The problem of type was very much in the air at that time. It was a question of finding intrinsic conditions for a simply connected Riemann surface to be hyperbolic or parabolic, that is to say conformally equivalent to a disk or the whole plane. At that time one would think of a Riemann surface primarily as spread out over the plane or the sphere, and there was a vague feeling that a surface would be hyperbolic if it was strongly branched and parabolic if there were few branch-points. Around 1930 Speiser had devised a scheme to describe some fairly simple Riemann surfaces by means of a graph and had written about it in his semiphilosophical style. Inspired by this rather crude idea Nevanlinna made a remarkable contribution both to the theory of Riemann surfaces and to the theory of meromorphic functions by showing that all surfaces with a finite number of logarithmic branch-points and no others can be completely described and classified as belonging to solutions of a differential equa- 
tion $S f=R(z)$ where $S f$ is the Schwarzian derivative and $R$ is a rational function. These Nevanlinna transcendentals are examples of meromorphic functions with given rational defects that add up to two. This whole approach is very classical in its beauty and simplicity, and one can say that in this way Nevanlinna entered the area of Riemann surfaces through a sidedoor.

Nevanlinna continues to study Riemann surfaces from the view-point of meromorphic functions for instance in his address to the International Congress in Zürich 1932. In that address there appears, probably for the first time, a clear reference to the second main theorem as a limiting case of the Hurwitz relation, an idea that had occupied Nevanlinna for some time, although only in rather vague form. One can also read between the lines and speculate that he may have been trying to solve the inverse defect problem, which as you know was finally solved by David Drasin only a few years ago.

I would nevertheless say that Nevanlinna's involvement with Riemann surfaces got its start only when he began to deal with arbitrary open Riemann surfaces, not by way of uniformization, but directly on the Riemann surface itself. In retrospect one easily forgets what a big step this was, and even if Nevanlinna was not alone his share in the development was definitely significant. In order to understand the general outlook at that time it is essential to remember that the theory of complex analytic manifolds did not exist, or was at best in its infancy, except for Hermann Weyl's justly famous "Die Idee der Riemannschen Fläche" which contained a correct modern definition of a Riemann surface as a one-dimensional complex manifold. This definition must have seemed a little too abstract to Nevanlinna, for he set out to give his own definition in more concrete terms.

What motivated him? For one thing, he spent part of the acedemic year 1938-39 in Göttingen and this alone may have inspired him to look back. I can think of another reason. "Eindeutige analytische Funktionen" is a masterpiece, but it is not selfcontained because it takes uniformization for granted. I can well imagine that Nevanlinna wanted to put his personal touch also to the theory of uniformization.

Whatever the reason, during this period he wrote several papers on Riemann surfaces, one in Crelle's Journal, one in Monatshefte, one in Göttinger Nachrichten, and one for Accademia d'Italia which was not published until 1943. I like especially the one for Accademia d'Italia. It is very elegantly written and introduces a new twist to the alternating method. One would probably have to agree that the mathematical content in these papers was not as interesting and innovative as one might have expected, but the mere fact that a mathematician of Nevanlinna's stature took up this almost dormant theory was definitely significant, and perhaps mostly because it caused Nevanlinna to think about these matters.

The war hit Finland in November 1939. Like so many others Nevanlinna was not in a position to devote himself to serious mathematics during and shortly after 
the war, with the possible exception of the Summer 1940 during the Finnish-Russian armistice. There are two important papers of Nevanlinna that were presented to the Finnish Academy of Science in 1940. The first, "Ein Satz über offene Riemannsche Flächen" is concerned with the type problem, but in the setting of arbitrary Riemann surfaces, and it is a direct outcome of Nevanlinna's occupation with the definition and construction of Riemann surfaces; his result is very simple, elegant, and of great generality. The second paper deals with square integrable differentials on Riemann surfaces, and it is much more than a routine paper. If my memory serves me right Nevanlinna told me the general idea of this paper already in the fall of 1939, before the outbreak of the war, but it is possible that the paper was actually written next summer. I like to discuss the impact of this particular paper on what later became known as the Classification Theory of Riemann surfaces. To what extent can Nevanlinna, together with Myrberg, be considered the founders of classification theory?

In an attempt to answer this question I find it convenient to set up a sort of time-table for the main events connected with the history of classification theory.

\begin{tabular}{|c|c|c|}
\hline Speiser & 1930 & graph of RS \\
\hline \multirow{2}{*}{ Nevanlinna } & 1930 & type problem \\
\hline & 1932 & transcendental functions \\
\hline Myrberg & 1933 & Green's function \\
\hline Hornich & 1933, 1935 & transc. hyperell. surfaces \\
\hline \multirow[t]{3}{*}{ Nevanlinna } & 1936 & Eind. anal. Funkt. \\
\hline & $1938-39$ & alternating method, zero bdry \\
\hline & 1940 & square integr. diff. \\
\hline Nevanlinna (Sario) & 1946 & Scand. Congr. \\
\hline \multirow[t]{2}{*}{ Sario } & 1948 & thesis \\
\hline & 1949 & C. R. notes \\
\hline Ahlfors & 1949 & extremal probl. on RS \\
\hline Ahlfors-Beurling & 1950 & conformal invariants \\
\hline Nevanlinna & 1953 & Uniformisierung \\
\hline Pfluger & 1957 & Theorie d. Riem. Fl. \\
\hline Ahlfors-Sario & 1960 & Riemann surfaces \\
\hline
\end{tabular}

Let me now comment on Nevanlinna's papers from the period 1938-1940. Through the concept of harmonic measure as applied to plane regions Nevanlinna came early to the distinction between regions with zero boundary (Nullrand) and positive boundary. This carries over at once to arbitrary open surfaces. He is aware that the surfaces with zero boundary are precisely the ones without a Green's function, and he quotes Myrberg who already 1933 had given the right definition of the Green's function and had proved that a Riemann surface which carries a 
non-constant bounded harmonic function has also a Green's function. Myrberg has a very ingenious proof which uses uniformization and also the convergence of the Blaschke product of a bounded analytic function. On the other hand, with harmonic measure this particular theorem becomes so trivial that Nevanlinna hardly needs to prove it, and at the same time he can show that the existence of a harmonic function with finite Dirichlet integral also implies the existence of a Green's function. Nevanlinna is also careful to mention Hornich for his work on transcendental hyperelliptic surfaces, which was rather similar to what Myrberg was also doing at approximately the same time.

In his paper on square integrable differentials Nevanlinna studies analytic differentials $\varphi d z$ such that $\int|\varphi|^{2} d x d y<\infty$. It is more or less trivial that they form a Hilbert space, but Nevanlinna wants to know to what extent the square integrable differentials are determined by their periods. This is the right question to ask, and he asks it for the right class of differentials. He proves, under the assumption that the surface has zero boundary, first of all that all periods over dividing cycles are automatically zero, and secondly that the differential is uniquely determined by its periods or, in other words, that a square integrable differential with zero periods is identically zero. He regards this as a property of surfaces with a null boundary in his sense, and at this point he does not raise the question whether this property would be shared by a wider class. From this point of view he does not need any other classification and no special notations. Nevertheless, I shall now adopt the notation that has become standard, namely Sario's $O$-notation.

This notation has a rather interesting history of its own. At some point, rather late in the game, Nevanlinna used the notation $N$ for the class of surfaces with null-boundary, now known as $O_{G}$. In my Commentarii paper of 1949 I picked up this notation and used $N_{1}$ for $O_{H D}$ (no Dirichlet bounded harmonic functions) and $N_{2}$ for $O_{A D}$ (no Dirichlet bounded analytic functions), and I made it clear that $N$ was short for Nevanlinna. On the other hand, in his book on uniformization theory Nevanlinna denotes these classes by $R_{H D}^{0}$ and $R_{A D}^{0}$. I tried to perpetuate the $N$-notation by using it again in my paper with Beurling, but to no avail. When it came to writing a book with Sario he reacted vehemently against the $N$-notation, precisely because of the connotation with Nevanlinna. I tried to argue with him, but to nobody's surprise he won out. Perhaps that was to the best for everybody, for there is now an accepted standard notation.

In the Ahlfors-Sario book on Riemann surfaces one finds an almost cabbalistic display of the inclusion relations between the various nullclasses of Riemann surfaces:

$$
O_{G} \subset O_{H P} \subset O_{H B} \subset{ }_{O_{A B}}^{O_{H D}} \subset O_{A D} .
$$

Here $O_{G}$ is the class of surfaces without Green's function, which is identical with 
the class of surfaces with "Nullrand" in Nevanlinna's terminology. The other notations are alsmost self-descriptive.

As I have already indicated Myrberg was the first to prove $O_{G} \subset O_{H P}$ while Nevanlinna proved $O_{G} \subset O_{H D}$ and probably $O_{H B} \subset O_{H D}$. Actually, $O_{H D}=O_{H B D}$ which makes most of the inclusions trivial. It is interesting that the corresponding equality $O_{A B}=O_{A B D}$ is very much harder and was not proved until 1979 by Sakai.

The question of strict inclusion was a hard nut to crack. After a false start (by myself) it was finally settled by Tôki who proved $O_{G}<O_{H B}<O_{H D}$. He also showed that there is no inclusion relation between $O_{H D}$ and $O_{A B}$.

I would like to say a little more about the role of Nevanlinna in this development. The Scandinavian Congress in Copenhagen 1946 marks Nevanlinna's return to mathematics after quite a while. He begins his congress talk with a review of Riemann surfaces, essentially as he had left it in 1940. Then he goes on to introduce some new results of his student, Sario. He acknowledges that Sario has studied the question of unique uniformization in "etwas allgemeinerer Fassung". The problem can be stated as follows: When is a plane region rigid in the sense that every conformal mapping on another plane region is given by a Möbius transformation. It had been proved by Koebe that the domain of discontinuity of a Schottky group has this property. To say that Sario's approach was only slightly more general is in my opinion an understatement. It is impossible for an outsider to assess the extent of a teacher-student relationship, but I have the impression that there must have been a lot of give and take between the two. There is strong evidence that Sario learned very much from Nevanlinna. For instance, Sario's "operator method" is essentially a very clever refinement of Nevanlinna's version of the alternating method. On the other hand, having been away from mathematics for so long Nevanlinna must have appreciated the opportunity to talk to a much younger gifted mathematician. If one reads between the lines in Nevanlinna's talk at the congress one can see that he is very impressed with Sario and he gives him full credit, although he could have done it a little more explicitly.

Sario, in his thesis, does not use the word "rigid", but uses the somewhat ambiguous term "hebbar" for the complement of a rigid region; it is never clear why just this property should make the complement removable. Actually, a region is rigid if and only if it is of class $O_{A D}$. This follows very easily from Schiffer's notation of span which he had introduced already in 1943, but this was not yet known in Europe due to the poor communications and lack of personal contact during the war. Sario's work shows that $O_{A D}$ is strictly larger than $O_{G}$ and he has a legitimate claim to have introduced the first nullclass which is demonstrably different from $O_{G}$. A little later, at the occasion of the next Scandinavian congress he introduces his $O$-notation and defines several classes of degeneracy. However, at that time the whole idea had already spread to several mathematicians, including myself, Bader and Parreau in France, and many others. 
In my paper in the Commentarii Helvetici, printed 1950 but presented 1949 , I had taken up the same kind of questions from the point of view of extremal problems on compact surfaces with boundary. As far as I can recall my inspiration for this work came directly from Myrberg and Nevanlinna, with both of whom I had corresponded, and also from Schiffer who was in Cambridge, Massachusetts, at that time. Although Nevanlinna in his paper on square integrable differentials does not mention $O_{H D}$ explicitly it is present in the background and the paper has been seminal in more ways than one because it asks just the right questions. For my part I do not hesitate to consider Myrberg and Nevanlinna the spiritual fathers of classification theory.

Everybody has blind spots, and in my Swiss paper I had a fixation on extremal problems. This prevented me from proving that there is a non-constant analytic function on every open Riemann surface, a problem which I believed to be open. Actually it had been solved in the affirmative by Behnke and Stein in connection with their extension of the Runge theorem to Riemann surfaces.

Let me now say something about Nevanlinna's book "Uniformisierung". It was written in 1952 and according to the preface it was based on lectures in Zürich and in Helsinki. Let me quote from a review that I wrote 1954:

"The problem of uniformization was in the center of mathematical interest in the beginning of this century. It was solved through the advancement of topology which led to the idea of the universal covering surface, and through the functiontheoretic discoveries of Poincaré and Koebe which permitted them to master the problem of conformal mapping. As is frequently the case the interest in the problem faded as soon as it was solved, but the methods to which it led continued to fascinate mathematicians up to this day.

It is thus not surprising that the topic of uniformization occupies very little space in the book of Nevanlinna, inspite of its title. What the reader finds is instead a modern monograph on Riemann surfaces. Such a book has long been missed in view of the surprising activity in this field during the last decades. Professor Nevanlinna is one of the chief instigators of this research, and it is therefore extremely fitting that he should be the first to present the results in book form."

Anything from Nevanlinna's pen is sure to be well planned and well written, and "Uniformisierung" is no exception. Nevertheless, it is not vintage Nevanlinna and it does not compare with his masterpiece "Eindeutige analytische Funktionen". Somehow I get the feeling that the publisher had commissioned this volume and that Nevanlinna wrote it reluctantly. Naturally, it served its purpose, but it did not make any big waves. It leaves the impression that the author was fed up with the subject, and as a matter of fact he never comes back to it. I speak with experience and compassion, for I felt much the same way when I had finished my book with Sario.

Nevanlinna's interest in Riemann surfaces did of course not go unnoticed by 
the younger Finnish mathematicians. Besides Sario, Lehto, Virtanen and Lokki all made important contributions. Here in Switzerland the work was continued mainly by Pfluger, whose book on Riemann surfaces came out in 1957. This is a beautiful and very readable book with many original results.

Beurling's and my paper on "Conformal invariants and small point sets" is a little earlier than Nevanlinna's book, and strictly speaking it does not deal with Riemann surfaces at all, for the simple reason that Beurling did not believe in Riemann surfaces. It came about because Beurling was visiting Harvard for the academic year 1948-49. It turned out that we had very similar ideas in common and the paper was the result of true and very fruitful cooperation. Its most important feature is that it contains the first codification of extremal length; I was very glad that Beurling consented to include it, for I was afraid it would otherwise never have been published. The conformal invariants appear as solutions of easily defined conformally invariant extremal problems. This comes close to my Commentarii paper, but there are many entirely new results. We used notations such as $M_{B}$, $M_{D}, M_{S B}, M_{S D}$ etc. ( $S$ stands for "schlicht") for the invariants and the corresponding null-classes were denoted by $N_{B}, N_{D}$, etc. The surprising thing is that many of these invariants turn out to be identical for reasons that are far from obvious.

The null-sets bring us to the second topic of this talk: small point sets in function theory. The connection with classification theory is obvious: the smallness of a closed set in the complex plane can be expressed through the properties of its complement regarded as a Riemann surface. Problems of this type are much older, one of the most typical being the Painleve problem: When is a set a removable singularity for the class of bounded analytic functions? It is natural that Nevanlinna would approach small point sets from the point of view of harmonic measure, and he did so much before his interest in general Riemann surfaces had awakened. He defines what he means by a set of harmonic measure zero, first with respect to a region, and then in the absolute sense. He recognizes that a set is of absolute harmonic measure zero if and only if it has zero capacity, a fact that was of course well known, but not exactly in Nevanlinna's sense.

When applied to the full boundary of a planar Riemann surface, which may be thought of as a plane region, this is of course another way of saying that a surface with null-boundary is one with no Green's function. But I must confess that I cannot follow Nevanlinna all the way when he tries to define sets of harmonic measure zero on the boundary of a Riemann surface, for instance on the boundary of a subregion of another Riemann surface. He does not have the notion of boundary element that would allow him to do so, and in fact he gets into a controversy with Teichmüller over this question. I don't want to take sides, especially since the whole question has become obsolete in view of the much more sophisticated definition of boundary first by R. H. Martin and later by Constantinescu-Cornea, Royden, Kuramochi, and many others. I apologize for not saying more about these refined 
compactification results, but it would take me rather far from Nevanlinna's sphere of influence.

Apart from these results the question of small point sets makes sense only in the plane, or by trivial generalization on surfaces of finite genus. For such surfaces the classes $O_{G}, O_{B}$ and $O_{D}$ are identical so that, for instance, sets of zero capacity are removable singularities for both bounded and Dirichlet bounded harmonic functions. On the other hand the removable sets for the Painlevé problem are those whose complement is of class $O_{A B}$.

At the early stage of the game considerable effort was spent on finding purely geometric characterizations of small point sets. Nevanlinna was quite interested in this question and had partial success in the case of zero capacity. He did not find any necessary and sufficient condition (and neither did I); this was done later by Erdös, Gillis, and Ursell. For Painlevé null-sets no necessary and sufficient conditions are known.

There is not much more to say about small point sets. For Nevanlinna, as for everybody else, they were part of the general classification theory, exemplified by the special case of harmonic measure. In Nevanlinna's writings there is a clear line showing how the problem of small sets has evolved from the old problem of type. As a point of some historical interest I should perhaps mention that there was a slightly new question connected with extremal length, namely the characterization of sets that are small enough not to influence the extremal length of a curve family. However, since Nevanlinna had no part in this development I shall not say more about it.

For different reasons I also want to say as little as possible about my book with Sario. In it Nevanlinna's influence is very noticeable because it comes from both sides. I hope it is a useful book as a serious introduction to Riemann surfaces, but it has been justly criticized for being too pedantic in some respects and too technical in others. I have learned my lesson never again to write a book with somebody else; it takes three times as long and the result leaves both authors unsatisfied. The meaty part of the book is the one that deals, once again, with extremal problems on compact Riemann surfaces with boundary, this time using the alternating method, or as Sario insisted to call it, the operator method. It does not go nearly as far as the book of Schiffer and Spencer on "Functionals of Riemann surfaces" (1954), but Schiffer's notion of span plays an important role in our book. In retrospect it seems a mistake not to have included the Bergman kernel function and the Bergman metric. We wanted to write a self-contained and reasonably elementary book; I don't know if we succeeded, but at least it has been rather widely read.

Our book was published 1960. A lot has happened after that, but without my participation. The Japanese have been very active; and so have Sario and his students. I do not want to dispute the importance of this work, but to my personal taste the classification craze has gone a little too far. Those who want to know 
more should read Sario-Nakai: Classification theory of Riemann surfaces, Springer Grundlehren 1970.

From Riemann surfaces Nevanlinna went to differential geometry, and I cannot say that I blame him. A change of pace is good for everybody, and I am sure he felt that he had said what he wanted to say about Riemann surfaces. Let me conclude by saying that the preparation of this paper has convinced me once more, if I needed it, that Nevanlinna's mathematics should be appreciated not only for its highest peaks but also for its very broad scope.

Harvard University

Department of Mathematics

Cambridge, Mass. 02138

USA

Received 5 March 1981 